\title{
Interdisziplinär und praxisbezogen: 11. Deutsche Kardiodiagnostik-Tage mit 12. Leipziger Symposium nichtinvasive kardiovaskuläre Bildgebung
}

Vom 21. bis 23. Februar 2019 finden die 11. Deutschen Kardiodiagnostik-Tage in Leipzig statt, zum 8. Mal als interdisziplinäre Veranstaltung unter der Ägide der AG Herz- und Gefäßdiagnostik der Deutschen Röntgengesellschaft (DRG) und der Arbeitsgruppen für CT und MRT der Deutschen Gesellschaft für Kardiologie (DGK) sowie erneut der AG Kardiovaskuläre Nuklearmedizin der Deutschen Gesellschaft für Nuklearmedizin (DGN). Ein
Interview mit Prof. Dr. Matthias Gutberlet und Prof. Dr. Holger Thiele, die zusammen mit Prof. Dr. Matthias Grothoff und PD Dr. Dr. Philipp Lurz die Tagung leiten.

Die Kardiodiagnostik-Tage finden 2019 zum 11. Mal statt - bereits zum 8. Mal auch in interdisziplinärer Zusammenarbeit zwischen der AG Herz- und Gefäßdiagnostik der DRG, der Arbeitsgruppen für CT und MRT der DGK sowie der AG Kardiovas- kuläre Nuklearmedizin der DGN. Welchen Mehrwert sehen Sie für die Teilnehmerinnen und Teilnehmer durch die interdisziplinäre Ausrichtung des Kongresses?

Prof. Dr. Holger Thiele: Aus meiner Sicht ist der große Mehrwert der unterschiedliche Blick auf die Krankheiten und die Pathologien aus der klinischen Sicht und der Sicht des Bildgebers. Das führt oft zu einer besseren Interpretation der Bildergebnisse und 




Prof. Dr. Holger Thiele

der daraus folgenden klinischen Empfehlung. Die Integration des klinischen Gesamtbildes des Patienten in die Bildinterpretation ist von zentraler Bedeutung für die Diagnose und Therapieempfehlung. Da können alle Fachdisziplinen am meisten von profitieren, wenn wir das gemeinsam machen.

Prof. Dr. Matthias Gutberlet: Ja, das ist eine unbedingte Voraussetzung für einen adäquaten Einsatz der Bildgebung in der Medizin, nicht nur im kardiovaskulären Bereich. Wir fassen die „interdisziplinäre“ Ausrichtung sogar noch weiter, indem wir den Kongress auch schon die letzten Jahre immer stärker multimodal ausgerichtet haben. Das heißt, wir gehen von der klinischen Fragestellung aus und betrachten, mit welchen bildgebenden Methoden wir die Diagnose am besten stellen und mit welchen wir die Therapieplanung und die finale Therapie am besten unterstützen können.

Sie wollen auch den klinischen Alltag modalitätenübergreifend in den Vordergrund rücken, d. h. Sie legen offensichtlich besonders viel Wert auf einen unmittelbaren Praxisbezug. Wie findet sich dieser Anspruch im Programm wieder?

Gutberlet: Ja, der Praxisbezug steht insbesondere am Donnerstag im Vordergrund des Programms. Hier werden zertifizierte Workshops für die Kardio-MRT und die Kardio-CT sowie ein Echo-Workshop und ein Kurs zur Nuklearkardiologie angeboten. Zusätzlich werden wir den Workshop zur MRT bei Patienten mit Schrittmachern und ICDs wiederholen, den wir 2018 zum ersten Mal angeboten hatten und der großen Zuspruch fand, da er auch für Kolleginnen und Kollegen geeignet ist, die sich bisher nicht mit der Kardio-MRT und Kardio-CT beschäftigen. Wichtig war auch die Erweiterung des Angebots für die MTRA mit den Kursen zur Zertifizierung als „Fachkraft für kardiovaskuläre Bildgebung" des VMTB, die es auch diesmal wieder geben wird. Ergänzt wird das Ganze noch durch fallbasierte Workshops, z. B. zur TAVI-Planung, Entzündungsdiagnostik oder zu angeborenen Herzfehlern.

Thiele: Den direkten Praxisbezug haben wir versucht auch durch die weitgehend paritätische Auswahl der Referenten zu gewährleisten, die sowohl aus der Radiologie als auch der Kardiologie kommen. Wichtig ist aber auch die Einbindung der verschiedenen Live-Fälle, die interventionelle Techniken in der kardiovaskulären Medizin mit der Bildgebung verknüpfen. Und dann haben wir ja auch noch für die wirkliche Praxis die Fallsammlungen, wo im Selbststudium oder unter Anleitung verschiedenste Fälle befundet werden können.

Mit den Kardiodiagnostik-Tagen wollen Sie zugleich Einsteiger und Experten ansprechen. Welche Programmpunkte könnten Sie hier jeweils empfehlen?

Thiele: Für die Einsteiger empfehle ich insbesondere die Kurse für CT und MRT oder Echo. Aber auch das Hauptprogramm bietet für Einsteiger oder Experten viele Informationen von der Basis bis zum aktuellen Stand der Wissenschaft.

Gutberlet: Korrekt. Für die Experten sind sicher die am Freitag und Samstag behandelten wissenschaftlichen Themen am interessantesten, so z. B. zu MINOCA am Freitag oder den neuen LAKE-LOUISE-CRITERIA-II und den neuen Ergebnissen der CT-Studien wie SCOT-HEART und PROMISE am Samstag. Auch die Sessions für spezielle Interessen, wie der TAVI-Workshop und der T1-T2Mapping-Workshop am Freitag, sind nicht nur für Einsteiger interessant.

Weshalb lohnt sich auch für Vertreter angrenzender Fächer wie der Herzchirurgie, Inneren Medizin, Allgemeinmedizin oder Nuklearmedizin ein Besuch?

Gutberlet: Neben der Kardiologie und der Radiologie sind alle diese Fächer an der Be-

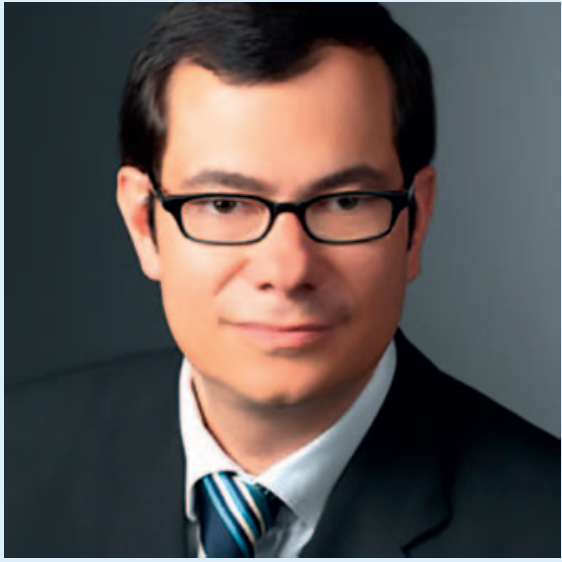

Prof. Dr. Matthias Gutberlet

handlung und Therapie von Patienten mit kardiovaskulären Erkrankungen beteiligt und müssen sich deshalb immer auf dem aktuellen Stand der Möglichkeiten der bildgebenden Diagnostik und Therapie halten, um die richtigen Methoden zum Wohle ihrer Patienten einsetzen zu können. Insofern „verschwimmen“ die Fachgrenzen hier ein wenig.

Thiele: Deshalb sind aus meiner Sicht die Fächer gar nicht so angrenzend. Sie sind ja alle integral für die Behandlung der Patienten, und die Bildgebung ist hier ein wesentlicher Baustein in der Diagnosestellung, Prognoseabschätzung, Therapieplanung und auch der Langzeittherapie. Ich hoffe, dass unser Programm allen Teilnehmenden etwas für den klinischen Alltag, aber auch für wissenschaftliche Fragestellungen bietet.

\section{Was bieten die Kardiodiagnostik-Tage speziell für MTRA?}

Thiele: Für die MTRA gibt es in diesem Jahr wieder einen speziellen MTRA-Kurs. Der hat sich in den letzten Jahren eine große Beliebtheit, einen großen Stellenwert erarbeitet.

Gutberlet: Neben den schon erwähnten modular aufgebauten Kursen I-III speziell für die MTRA, die zur Hälfte auch von MTRA für MTRA gehalten werden, sind auch die Live-Demos am Donnerstag, die zwischen den Kursen für MTRA und Ärzten liegen, genauso wie die CT- und MRT-FaceOff-Sessions am Freitag, wo die verschiedenen Firmen ihre verschiedenen Softwareangebote fallbasiert demonstrieren, in das Kursangebot für MTRA integriert. 
Gibt es Veranstaltungen, die sich bereits in den vergangenen Jahren als „Publikumsmagneten“ etabliert haben und 2019 eine Fortsetzung erfahren?

Gutberlet: Das sind auf jeden Fall die 3 LiveDemos am Donnerstag zwischen den Workshops, bei denen sowohl die Bildgebung, MRT und CT, als auch die daran anschließende bildgestützte Therapie live demonstriert werden. Dadurch wird es besonders klar, welchen Stellenwert die einzelnen Methoden für die bildgestützte Therapie, wie z. B. die TAVI, haben.

Thiele: Ein Publikumsmagnet waren und sind immer die bereits genannten „Face-offSessions“, in denen verschiedene SoftwareAnbieter live die Performance ihrer Software zum Post-Processing der von uns gestellten MRT- oder CT-Bilder darstellen. Das ist sehr interaktiv und hat auch einen gewissen, aber immer fairen Wettkampfcharakter. Beliebt sind aber auch viele andere Sitzungen, wie die Sitzung zu den größten Fehlern, die ausgewiesene Experten in der Bildgebung gemacht haben.

Traditionell ehren Sie im Rahmen der offiziellen Eröffnung einen Vertreter/eine Vertreterin aus der Kardiologie oder Ra- diologie, der oder die sich um die kardiovaskuläre Bildgebung besonders verdient gemacht hat. Können Sie schon verraten, wem diese Ehrung 2019 zuteilwird?

Thiele: In diesem Jahr ist es einmal kein Kardiologe oder Radiologe! Wir haben uns für Dr. Bernhard Schnackenburg ausgesprochen, der seit vielen Jahrzehnten die Entwicklung der kardialen MRT als Physiker vorangebracht hat. Herausragend ist sicherlich seine Fähigkeit, uns Mediziner für die Physik der MRT zu begeistern und vor allem verständlich zu erklären. Ich habe selbst erst durch Dr. Schnackenburg viele der Sequenzen verstanden. Er hat ganz wesentlich zur klinischen Nutzbarkeit und Alltagstauglichkeit der kardialen MRT beigetragen. Für dieses Lebenswerk wird Dr. Schnackenburg geehrt, was er mehr als verdient hat.

Gutberlet: Auch ich habe eine sehr persönliche Beziehung zu unserem nächsten Laureaten. Er hat mir damals noch an meiner radiologischen Ausbildungsstätte, dem Virchow-Klinikum der Charité in Berlin, als Weiterbildungsassistent MR-Physik beigebracht, wovon ich noch heute profitiere. Dort haben wir uns so manche Abende oder Wochenenden gemeinsam im MRT-Labor mit Messungen „um die Ohren geschlagen“. Des Weiteren kenne ich kaum einen MR-Physiker, der sich so gut mit dem Herzen auskennt wie er.

Abschließend noch ein Frage: Haben Sie schon Programm-Highlights identifizieren können, auf die Sie sich persönlich besonders freuen?

Gutberlet: Neben der Ehrung für das Lebenswerk von Dr. Schnackenburg freue ich mich vor allem auf lebhafte Diskussionen in allen Programmpunkten, weshalb wir versucht haben, überall dafür genügend Zeit einzuräumen und deshalb die Redezeit der einzelnen Referenten bewusst etwas knapper gehalten haben.

Thiele: Ich freue mich immer wieder auf die Debatten, welche Methode für die einzelne Fragestellung die beste ist. Da sind die einzelnen Experten für ihren Bereich gefragt, am Ende finden dann aber alle meist einen guten Weg, wie man im Alltag das Beste aus den einzelnen Methoden herausholen kann. Aber nur eine Sache hervorzuheben würde dem Gesamtprogramm nicht gerecht werden. Aus meiner Sicht ist alles spannend.

\section{Vielen Dank für das Gespräch!}

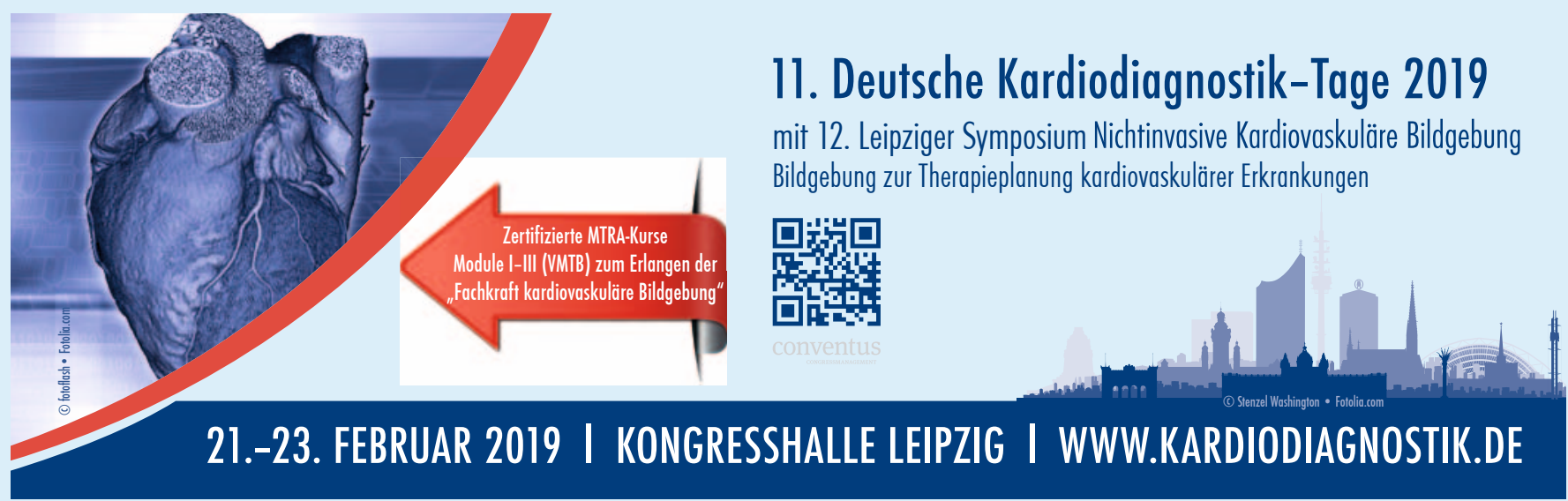

